\title{
Extralimital triradii as a putative marker of schizotypy
}

By: James T. Chok and Thomas R. Kwapil

Chok, J.T., \& Kwapil, T.R. (2005). Extralimital triradii as a putative marker of schizotypy. Schizophrenia Research, 76, 239-245. http://dx.doi.org/10.1016/j.schres.2004.12.015

\section{Made available courtesy of Elsevier: http://www.elsevier.com}

\author{
*** Reprinted with permission. No further reproduction is authorized without written \\ permission from Elsevier. This version of the document is not the version of record. Figures \\ and/or pictures may be missing from this format of the document. ***
}

\begin{abstract}
:
Dermatoglyphic anomalies are reported to occur at a higher rate in schizophrenic patients and schizotypic individuals than in the general population, supporting the hypothesis that they are a marker of vulnerability for such conditions. Dermatoglyphic anomalies are hypothesized to indicate severe disruptions in the second trimester of prenatal development, a time period that appears to be etiologically relevant to the development of schizophrenia and related conditions. The present study provides the first examination of extralimital triradii in schizotypic young adults ( $n=197)$ and control participants $(n=135)$ identified by the Revised Social Anhedonia Scale [Eckblad, M., Chapman, L.J., Chapman, J.P., Mishlove, M., 1982. The Revised Social Anhedonia Scale. Unpublished test (copies available from T.R. Kwapil, Department of Psychology, University of North Carolina at Greensboro P.O. Box 26164 Greensboro, NC 27402-6164)], the Perceptual Aberration Scale [Chapman, L.J., Chapman, J.P., Raulin, M.L., 1978. Body image aberration in schizophrenia. J. Abnorm. Psychol. 87, 399-407], the Magical Ideation Scale [Eckblad, M.L., Chapman, L.J., 1983. Magical ideation as an indicator of schizotypy. J. Consult. Clin. Psychol. 51, 215-225], and the Physical Anhedonia Scale [Chapman, L.J., Chapman, J.P., Raulin, M.L., 1976. Scales for physical and social anhedonia. J. Abnorm. Psychol. 85, 374-382]. As hypothesized, the schizotypic participants (6.6\%) exhibited significantly higher rates of extralimital triradii than control participants (1.5\%). These findings strongly encourage the future investigation of extralimital triradii in at-risk and psychotic populations.
\end{abstract}

Keywords: extralimital | triradii | schizotypy | dermatoglyphic | schizophrenia | psychology

Article: 


\section{Introduction}

\subsection{Dermatoglyphic anomalies, schizophrenia, and schizotypy}

Dermatoglyphic anomalies, which include disruptions in ectodermic landmarks on the fingers and palm, as well as asymmetry between such landmarks on homologous fingers of the two hands, are presumed to indicate disruption in cell migration during the second trimester of prenatal development. Consistent with neurodevelopmental hypotheses of schizophrenia (e.g., Andreason, 1999), dermatoglyphic anomalies have been widely reported to occur at a heightened rate in patients diagnosed with the disorder (e.g., [Fananas et al., 1996], [Kemali et al., 1976], [Markow and Wandler, 1986] and [Mellor, 1992]). Current models of schizophrenia suggest that neurodevelopmental vulnerability is expressed across a dynamic continuum of schizophreniclike expression, referred to as schizotypy. This continuum ranges from relative health to subclinical deviance to schizophrenia-spectrum personality disorders to full-blown clinical psychosis. Such models suggest that schizotypic individuals should exhibit cognitive and biobehavioral deficits that are similar, albeit less frequent and severe, to those seen in schizophrenia. In line with this idea, recent studies have reported higher rates of dermatoglyphic anomalies in patients with schizotypal personality disorder (Weinstein et al., 1999) and in nonpsychotic young adults identified by psychometric measures of schizotypy ( [Chok et al., 2005] and [Rosa et al., 2000]). Rosa et al. (2000) and Barrantes-Vidal et al. (2002) found that fluctuating dermatoglyphic asymmetry, which is thought to reflect prenatal developmental instability, was more prevalent in young adolescents scoring high on the Social Anhedonia and Physical Anhedonia Scales (measures of negative schizotypy) in comparison to adolescents with high scores on the Perceptual Aberration Scale (a measure of positive schizotypy) and control participants. These findings are consistent with evidence that individuals with prominent negative symptoms of schizotypy and schizophrenia exhibit more neurodevelopmental abnormalities than individuals with predominantly positive symptoms ( [Murray et al., 1992] and [van Os et al., 1998]). Chok et al. (2005) assessed 12 different anomalies in college-aged students and found that individuals scoring high on the Perceptual Aberration/Magical Ideation Scales (measures of positive schizotypy) exhibited reduced total finger ridge count and less complex pattern types than control participants (they did not evaluate individuals scoring high on the negative schizotypy scales). Fananas et al. (1996) examined a pair of monozygotic twins discordant for schizotypy reporting reduced total finger ridge count and the presence of ridge dissociation in the affected twin, but not the discordant twin. These findings were consistent with Torrey et al. (1994) who examined dermatoglyphic anomalies in twins discordant for schizophrenia. In contrast, Turek (1990) found increased total finger ridge count in patients with schizophrenia in comparison to controls. In sum, the majority of studies investigating dermatoglyphics in patients with schizophrenia and individuals scoring high on schizotypy have revealed heightened rates of dermatoglyphic anomalies among affected individuals. Taken together with investigations examining other neurodevelopmental abnormalities associated with 
schizophrenia and schizotypy, it appears that markers of maldevelopment occur across symptom dimensions, but are more prevalent in those exhibiting elevated negative symptoms.

\subsection{Extralimital triradii}

The present study extends the work of Chok et al. (2005) by examining the presence of extralimital triradii in a sample of schizotypic young adults. Extralimital triradii are rare anomalies that occur when a triradius, a common dermatoglyphic landmark characterized by the confluence of three ridges, would have formed had there been a larger area of ridged skin on the finger. In essence, the pattern is "too big" for the finger and the triradius, or triradii, is not visible because it did not fit on the ridged area of the finger tip. Extralimital triradii are almost exclusively the result of very large whorl patterns (Schaumann and Alter, 1976). Fig. 1 presents a normally developed print and a print with two extralimital triradii. Holt (1968) estimated that extralimital triradii only occur in approximately $0.7 \%$ of females and $1.4 \%$ in males in the British population. This anomaly can be quickly and easily identified when examining fingerprints, adding to its attractiveness as a potential marker for schizophrenia and related disorders. Unlike other dermatoglyphic anomalies, the relationship between extralimital triradii and psychopathology has not been explored. A search of "extralimital" on the National Library of Medicine search engine, PubMed, yielded only two publications relevant to dermatoglyphics — each a case study of a child with a severe medical condition who exhibited an extralimital triradius ( [Krush et al., 1988] and [White and Marx, 1978]). 


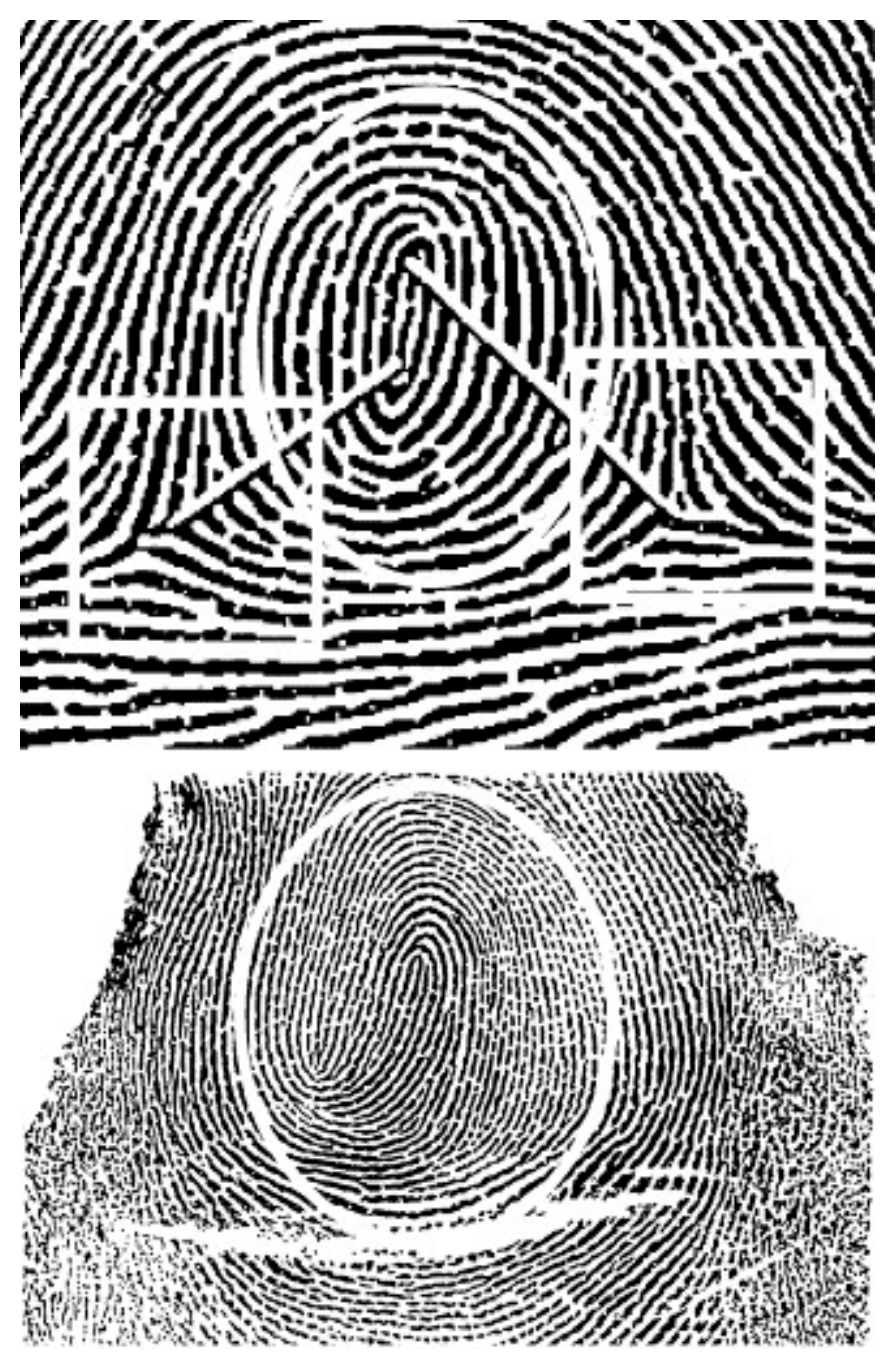

Fig. 1. Top figure: fingerprint with whorl pattern and observable triradii. The oval displays the whorl pattern, while the squares indicate normally appearing triradii. The straight lines connect the core of the whorl with the triradii. Bottom figure: fingerprint with extralimital triradii. The oval displays a double-loop whorl pattern. Neither of the expected triradii appear within the ridged surface of the print. The unusual size of the print is reflected by the fact that it extends below the flexion crease in the finger. Adapted from Holt (1968).

Studies examining embryonic ridge development have provided several insights that may help explain the occurrence of extralimital triradii. For example, the development of ridges occurs on volar pads on the fingers, palms, and soles of the foot. The volar pads on the hands are soft, bulbous areas that swell rapidly between 6.5 and 10.5 weeks of fetal development in humans, and then regress thereafter, until they reach a size similar to their size at birth. As the pads begin the regression process, the force and pressure that occurs contribute to ridge development. By the 
24th week, ridges have replaced the volar pads and fingerprint patterns resemble adult formations. Babler (1978) provided evidence that the timing of volar pad involution is related to the type of ridge patterns that develop, with early ridge formation being associated with whorl patterns, intermediate ridge formation associated with the development of loop patterns, and late ridge development associated with arches. In essence, more complex patterns occur earliest and patterns become less complex the later ridge formation occurs. Other investigations have cited that fetal edema, or excess fluid in the epidermis, can lead to larger volar pads, which require a greater number of ridges to cover them during the regression process (Schaumann and Alter, 1976). Penrose (1967) provided evidence that individuals with Turner's syndrome, who often display edema at birth, have increased ridge counts. Moreover, given that the frequency of whorl patterns, which yield the largest ridge counts, was similar between patients with Turner's syndrome and control participants, the higher ridge count total was attributable to more ridges per pattern rather than complexity of the pattern. Taken together, these insights into embryonic dermatoglyphic development suggests that if an increased incidence of extralimital triradii are present in schizotypic individuals, prenatal insults may be occurring early in the ridge formation process (i.e., during weeks 11 and 12 of fetal development). In addition, the instability arising from these insults may be characterized by excess fluid availability, which allows for the growth of more complex dermatoglyphic patterns, such as whorls and extralimital triradii.

\subsection{Goals and hypotheses of the present study}

The current study examined the presence of extralimital triradii in psychometrically identified schizotypic young adults and control participants recruited from a college student sample. While college graduates exhibit a slightly lower prevalence of schizophrenia than the general population (Robins et al., 1984), they provide an appropriate sample for a preliminary examination of the relationship of schizotypy with dermatoglyphic anomalies. Specifically, longitudinal studies have reported that psychometrically identified schizotypic college students are at heightened risk for developing psychotic disorders and schizophrenia-spectrum illnesses (e.g., [Chapman et al., 1994] and [Kwapil, 1998]), while Chok et al. (2005) reported that higher rates of dermatoglyphic anomalies are found in such samples. In the present study, it is hypothesized that schizotypic participants will exhibit a higher rate of extralimital triradii than control participants. In addition, given previous findings of dermatoglyphic anomalies in patients with schizophrenia and individuals with schizotypy, it is hypothesized that individuals with schizotypy will exhibit an increased rate of extralimital triradii, and that individuals with high negative schizotypy (i.e., elevations on the Social Anhedonia Scale and/or Physical Anhedonia Scale) will exhibit a greater number of anomalies. Consistent with the findings of a previous study investigating pattern type and schizotypy (Chok et al., 2005), it is predicted that individuals scoring high on the schizotypy scales will also exhibit fewer whorls than control participants. Lastly, since extralimital triradii occur almost exclusively on whorl patterns, it is 
assumed that the subgroup of individuals exhibiting extralimital triradii will naturally exhibit more whorls than other participants.

Such findings would support further study of the extent to which such anomalies are associated with schizotypic psychopathology and increased risk for developing schizophrenia-spectrum disorders. According to Garver (1987), if dermatoglyphic anomalies are robust markers, they should be apparent at all stages of the disorder. Thus, the initial step is to determine whether schizotypic individuals exhibit increased rates of severe dermatoglyphic anomalies in comparison to control participants.

\section{Method}

\subsection{Participants}

Dermatoglyphic anomalies were assessed in college students identified by scores on the Perceptual Aberration (Chapman et al., 1978), Magical Ideation (Eckblad and Chapman, 1983), Revised Social Anhedonia (Eckblad et al., 1982) and Physical Anhedonia (Chapman et al., 1976) Scales administered in general psychology courses at the University of North Carolina at Greensboro between Spring 2001 and Fall 2002. Participants who received standard scores of at least 1.96 on any of the scales and control participants who received standard scores of less than +0.5 on each of the scales were invited to participate in the dermatoglyphic assessment. The participants were limited to Caucasian and African-American students because normative data on the schizotypy scales are not available for other ethnic groups. The final sample consisted of 197 schizotypic and 135 control participants. The groups did not differ significantly on age, years of education, or gender and ethnic composition.

\subsection{Schizotypy questionnaires}

The Perceptual Aberration Scale consists of 35 items that tap schizotypal perceptual experiences and bodily distortions. The Magical Ideation Scale is made up of 30 items that measure belief in implausible or invalid causality. The Revised Social Anhedonia Scale consists of 40 items that tap asociality and indifference to others. The Physical Anhedonia Scale includes 61 items that measure deficits in sensory and aesthetic pleasure. The questionnaires have good psychometric properties as summarized by Chapman et al. (1995). The items from the schizotypy scales were intermixed with a 13-item infrequency scale (Chapman and Chapman, 1983) that was designed to screen out participants who responded in a random or "fake-bad" manner. Participants who endorsed three or more items on the infrequency scale were not included in the dermatoglyphic assessment. 


\subsection{Dermatoglyphic assessment}

An extralimital triradius was defined as being present when a finger pattern extended beyond the ridged portion of the skin. Fingerprints were made by one graduate and four undergraduate researchers using Identicator Perfect Ink printing apparatus (www.identicatorinc.com). The researchers were unaware of participants' group membership throughout the study. Prints were scored by the principal investigator.

\subsection{Procedure}

Candidate schizotypic and control participants were contacted by telephone or email and invited to participate in the dermatoglyphic assessment. Ninety-two percent of the students contacted agreed to participate in the study. The participation rate did not differ across the groups. Participants completed the dermatoglyphic assessment within 7 weeks of the mass screening assessment. Participants received course credit or payment for completing the study.

\section{Results}

Extralimital triradii were exhibited by 13 of the schizotypic participants (6.6\%) and two of the control participants (1.5\%), Fisher's Exact test (two-sided) $=0.03$. Consistent with Holt (1968), extralimital triradii were more common in male participants (8.2\%) than female participants (3.2\%) for the entire sample, Fisher's Exact test (two-sided) $=0.07$. The odds ratio was 4.70 (95\% confidence interval 1.16-18.90). As predicted, individuals with schizotypy as a group exhibited fewer whorls than control participants. Also consistent with our hypotheses, extralimital triradii occurred exclusively on whorl patterns and the presence of extralimital triradii was related to the number of whorl patterns ( $\mathrm{rpb}=0.31, \mathrm{p}<0.05)$. Table 1 displays the number of extralimital triradii found according to digit type. Given that some participants had more than one digit with an extralimital triradius, the sum of the numbers in this table is 19. Extralimital triradii occurred most frequently on the ring finger and never occurred on the little finger. Table 2 contains the number of participants exhibiting extralimital triradii according to schizotypy dimension. Individuals scoring high on only the Perceptual Aberration/Magical Ideation Scales were characterized as having positive schizotypy. Participants scoring high on the Physical Anhedonia and/or the Social Anhedonia Scale were considered to have negative schizotypy, and participants with elevations on both dimensions were considered to have a mixed presentation of schizotypy. Consistent with our predictions, extralimital triradii were more likely to occur in students with high negative schizotypy (69\% of the individuals with extralimital triradii had elevated negative schizotypy scores). 


\begin{tabular}{|c|c|c|c|c|}
\hline Right thumb & Right index & Right middle & Right ring & Right little \\
\hline 2 & 2 & 2 & 4 & 0 \\
\hline Left thumb & Left index & Left middle & Left ring & Left little \\
\hline 1 & 3 & 1 & 4 & 0 \\
\hline
\end{tabular}

\section{Table 2. Number of extralimital triradii by dimension of schizotypy \\ Positive schizotypy Negative schizotypy Mixed schizotypy \\ $\begin{array}{lll}4 & 8 & 1\end{array}$}

Additional data regarding dermatoglyphic anomalies was available for a subset of participants $(\mathrm{n}=199)$. Extralimital triradii were associated with total finger ridge count $(\mathrm{rpb}=0.37, \mathrm{p}<0.01)$, absolute finger ridge count $(\mathrm{rpb}=0.42, \mathrm{p}<0.01)$ and fluctuating dermatoglyphic asymmetry for total finger ridge count $(\mathrm{rpb}=0.18, \mathrm{p}<0.05)$ and absolute finger ridge count $(\mathrm{rpb}=0.26, \mathrm{p}<0.01)$. Extralimital triradii were not significantly associated with a-b ridge count, ridge dissociation, atd angle, and fluctuating dermatoglyphic asymmetry of the a-b ridge count and atd angle.

\section{Discussion}

The current study investigated the presence of a previously unstudied dermatoglyphic anomaly in a sample of psychometrically identified schizotypic college students. Consistent with a previous investigation of schizotypy and pattern type, individuals with schizotypy displayed fewer whorls than control participants. In addition, the main hypothesis of this study was supported as schizotypic individuals displayed a significantly higher rate of extralimital triradii than control participants. This finding strongly encourages the examination of these severe anomalies in patients with schizophrenia and at-risk populations. Until now, extralimital triradii have never been incorporated into an empirical investigation attempting to link dermatoglyphic anomalies and psychopathology. However, this anomaly occurred almost 4.5 times more often in schizotypic than in control participants (and almost seven times more often in the schizotypic group than in the general population estimate by Holt, 1968). Extralimital triradii were examined because they can be collected and scored quickly (approximately 10 min per participant) and analyzed on an individual level, making this potential marker an attractive measure of risk for schizotypy. Barring any severe environmental damage to the ectoderm, this marker is also impenetrable to postnatal developmental changes that may occur throughout the course of the development of schizophrenia. However, the lack of study of extralimital triradii in psychopathology research limited making hypotheses beyond the general expectation of heightened rates of the anomaly in schizotypy (and suggests that our findings should be 
considered exploratory or hypothesis-generating in nature). These preliminary findings encourage us to examine the relationship of extralimital triradii with other clinical and neurocognitive measures of schizotypic deficits. It should be noted that the present study did not assess finger size. Future studies should examine the relationship between finger size and the presence of extralimital triradii in order to determine the relationship between these variables. One might conjecture that individuals with smaller digits would be more likely to exhibit extralimital triradii given that they are oversized whorl patterns. Conducting clinical interviews with individuals with extralimital triradii, as well as with individuals who exhibit other dermatoglyphic anomalies, and tracking their progress through follow-up interviews may prove to be useful for improving our understanding of the role of prenatal insults as a moderator of risk for schizophrenia. In addition, establishing the rate of this anomaly in patients with schizophrenia may also be useful for determining the impact of prenatal insults in the development of schizophrenia.

\section{References:}

N.C. Andreason, 1999. A unitary model of schizophrenia: Bleuler's fragmented phrene as schizencephaly. Arch. Gen. Psychiatry, 56 (1999), pp. 781-787

W.J. Babler, 1978. Prenatal selection and dermatoglyphic patterns. Am. J. Phys. Anthropol., 48 (1) (1978), pp. 21-27

N. Barrantes-Vidal, L. Fananas, A. Rosa, B. Capparos, M.D. Riba, J.E. Obiols, 2002. Neurocognitive, behavioural and neurodevelopmental correlates of schizotypy clusters in adolescents from the general population. Schizophr. Res., 61 (2002), pp. 293-302

Chapman, L.J., Chapman, J.P., 1983. Infrequency Scale for Personality Measures. Unpublished scale available from T.R. Kwapil, Department of Psychology, University of North Carolina at Greensboro, Greensboro, NC 27402.

L.J. Chapman, J.P. Chapman, M.L. Raulin, 1976. Scales for physical and social anhedonia. J. Abnorm. Psychol., 85 (1976), pp. 374-382 
L.J. Chapman, J.P. Chapman, M.L. Raulin, 1978. Body image aberration in schizophrenia. J. Abnorm. Psychol., 87 (1978), pp. 399-407

L.J. Chapman, J.P. Chapman, T.R. Kwapil, M. Eckblad, M.C. Zinser, 1994. Putatively psychosis-prone subjects 10 years later. J. Abnorm. Psychol., 103 (1994), pp. 171-183

J.P. Chapman, L.J. Chapman, T.R. Kwapil, 1995. Scales for the measurement of schizotypy. A. Raine, T. Lencz, S. Mednick (Eds.), Schizotypal Personality Disorder, Cambridge Univ. Press, Cambridge, England (1995)

J.T. Chok, T.R. Kwapil, A. Scheuermann, 2005. Dermatoglyphic anomalies in psychometrically identified schizotypic young adults. Schizophr. Res., 72 (2-3) (2005), pp. 205-214

M.L. Eckblad, L.J. Chapman, 1983. Magical ideation as an indicator of schizotypy. J. Consult. Clin. Psychol., 51 (1983), pp. 215-225

Eckblad, M., Chapman, L.J., Chapman, J.P., Mishlove, M., 1982. The Revised Social Anhedonia Scale. Unpublished test (copies available from T.R. Kwapil, Department of Psychology, University of North Carolina at Greensboro P.O. Box 26164 Greensboro, NC 27402-6164).

L. Fananas, J. Van Os, C. Hoyos, J. McGrath, C.S. Mellor, R. Murray, 1996. Dermatoglyphic a$\mathrm{b}$ ridge count as a possible marker for developmental disturbance in schizophrenia: replication in two samples. Schizophr. Res., 20 (1996), pp. 307-314

D.L. Garver, 1987. Methodological issues facing the interpretation of high-risk studies: biological heterogeneity. Schizopr. Bull., 13 (3) (1987), pp. 525-529

S.H. Holt, 1968. The Genetics of Dermal Ridges. Charles C. Thomas, USA (1968) 
D. Kemali, N. Polani, P.E. Polani, A. Amati, 1976. A dermatoglyphic study of 219 Italian schizophrenic males. Clin. Genet., 9 (1) (1976), pp. 51-60

A.J. Krush, B.A. Schaumann, H. Youssoufian, 1988. Arachnodactyly and unusual dermatoglyphics: study of a case. Am. J. Med. Genet., 31 (1) (1988), pp. 57-62

T.R. Kwapil, 1998. Social anhedonia as a predictor of the development of schizophreniaspectrum disorders. J. Abnorm. Psychol., 107 (1998), pp. 558-565

T.A. Markow, K. Wandler, 1986. Fluctuating dermatoglyphic asymmetry and the genetics of liability to schizophrenia. Psychiatry Res., 19 (1986), pp. 323-328

C.S. Mellor, 1992. Dermatoglyphic evidence of fluctuating asymmetry in schizophrenia. Br. J. Psychiatry, 160 (1992), pp. 467-472

R.M. Murray, E. O'Callaghan, D. Castle, S.W. Lewis, 1992. A neurodevelopmental approach to the classification of schizophrenia. Schizophr. Bull., 18 (1992), pp. 318-332

L.S. Penrose, 1967. Finger-print pattern and the sex chromosomes. Lancet, 1 (7485) (1967), pp. 298-300

L.N. Robins, J.E. Helzer, M.W. Myrna, H. Orvaschel, E. Gruenberg, J.D. Burke, A.R. Darrel, 1984. Lifetime prevalence of specific psychiatric disorders in three sites. Arch. Gen. Psychiatry, 41 (1984), pp. 949-958

A. Rosa, J. van Os, L. Fananas, N. Barrantes, B. Caparros, B. Gutierrez, J. Obiols, 2000.

Developmental instability and schizotypy. Schizophr. Res., 43 (2000), pp. 125-134 
B. Schaumann, M. Alter, 1976. Dermatoglyphics in Medical Disorders. Springer-Verlag, New York (1976)

E.F. Torrey, E.H. Taylor, H.S. Bracha, A.E. Bowler, T.F. McNeil, R.R. Rawlings, P.O. Quinn, L.B. Bigelow, K. Rickler, K. Sjostrom, E.S. Higgins, I.I. Gottesman, 1994. Prenatal origin of schizophrenia in a subgroup of discordant monozygotic twins. Schizophr. Bull., 20 (1994), pp. $423-431$

S. Turek, 1990. Dermatoglyphics and schizophrenia: analysis of quantitative traits. Coll. Antropol., 14 (1) (1990), pp. 137-150

J. van Os, P. Jones, P. Sham, P. Bebbington, R.M. Murray, 1998. Risk factors for onset and persistence of psychosis. Soc. Psychiatry Psychiatr. Epidemiol., 33 (1998), pp. 596-606

D.D. Weinstein, D. Diforio, J. Schiffman, E. Walker, R. Bonsall, 1999. Minor physical anomalies, dermatoglyphic asymmetries, and cortisol levels in adolescents with schizotypal personality disorder. Am. J. Psychiatry, 156 (4) (1999), pp. 617-623

B.J. White, S.J. Marx, 1978. Dermatoglyphic and radiographic findings in a mother and daughter with pseudohypoparathyroidism. Clin. Genet., 13 (4) (1978), pp. 359-368 